\title{
Design of Low Power Current Mode Square-Root Circuit
}

\author{
Bindu Thakral $^{\mathrm{a}}$ \\ ${ }^{a}$ Assistant Professor, Electronics and Communication Engineering Department, Ansal University, Gurgaon, \\ India
}

\begin{abstract}
Analog circuits have been viewed as a voltage dominated type of signal processing. With the advent of CMOS technology, shrinking feature size, and reduction of supply voltage a new class of circuits developed: currentmode circuits. Among current-mode circuits "Translinear circuits" is one of the important and emerging research interests for low power analog designers. It is the practical means of implementing nonlinear signal processing functions. The performance of analog circuits strongly depends upon techniques used to exploit the characteristics of the transistors. In this paper, using exponential current-voltage relationship of MOS transistor when biased in subthreshold-mode, a square-root circuit is proposed. The circuit simulations were carried on TSMC 0.18 micrometer technology using ELDO Simulator.
\end{abstract}

Index Terms: Weak inversion, Translinear principle, Square-root, Square circuit.

(C) 2015 Published by MECS Publisher. Selection and/or peer review under responsibility of the Research Association of Modern Education and Computer Science.

\section{Introduction}

New electronics for medical monitoring promise low-cost, maintenance-free, and lightweight devices which are critical in long-term medical measurements and in home-based tele-monitoring services. The translinear principle proposed by Barry Gilbert in 1975 is one of the important contributions to circuit theory in the electronics era [1]. This is simply a design principle that exploits conservation of energy (KVL) around circuit loops which have specific topological properties. Translinear circuit interpretation stems from the fact that the transconductance of a BJT transistor is linearly proportional to its collector current.

In other way, it is a physical device whose transconductance and output current through the device have linear relation between them, i.e. output current is exponentially dependent to the controlling voltage with the condition that the controlling voltage must exhibit diode behavior. Such circuits were originally developed for nonlinear analog signal processing, with inputs and outputs in the form of currents. Using current mode circuits, complex mathematical functions can be easily designed that are not possible with the standard linear analog

\footnotetext{
* Corresponding author. Tel.: +91-9871003951

E-mail address: binduthakral@ansaluniversity.edu.in
} 
building blocks. Most of the work on translinear circuits to date uses bipolar transistors and the emphasis is on high precision and high speed. One fascinating aspect of Translinear circuits are their insensitivity to isothermal temperature variations. Mostly translinear building blocks have been designed to work in the low MHz range.

With advances in high speed analog-to-digital converter (ADC) and digital-signal-processing (DSP) technologies, most complex high-frequency signal processing can be done in the digital domain. Another exciting research area that evolved in last few years, is the synthesis of analog VLSI for sensory information processing systems [2,3] employing MOS transistors operating in subthreshold region [4, 5]. To exploit translinear behavior, MOSFET is made to operate in subthreshold region and has wide application in analog neuromorphic LSI and VLSI systems [6-9].

For subthreshold MOSFET

$$
\begin{aligned}
& I_{D S}=I_{o} e^{\left(k V_{G}-V_{S}\right) / V_{T}} \\
& g_{m}=\frac{d}{d V_{G}} I_{D S}=\frac{k}{V_{T}} I_{D S}
\end{aligned}
$$

In this paper, current-mode translinear circuit square-root is implemented using subthreshold MOSFET. The working of these circuits is based on generalized translinear principle valid at all inversion level. A squarerooting circuit has been found widely in instrumentation and measurement systems. For example, it can be used to linearize a signal from a differential pressure flow meter, or to calculate the r.m.s. value of an arbitrary waveform [10]. Typically, voltage-mode square-rooting circuits can be realized by using operational amplifiers (op-amps) and attached to passive and active elements such as an analog multiplier to form squarer and resistors [11], the BJT to form a log and antilog amplifier [12], MOS transistor operating in triode region [13]. However, op-amp-based square-rooting circuit the high frequency limitation due to the finite gain bandwidth product of the op-amp and some of these not suitable for IC implementation. The current-mode square-root circuit has wide application in design of filters $[14,15]$.

The proposed square-rooting circuits yield the following advantages:

(i) The square-rooting circuit of $[16,17]$ are limited the high-frequency due to the finite GBW of opamp or MOS transistor operated in non-saturation whereas the proposed circuits without these restrictions.

(ii) The proposed square-rooting circuit is more operate high-frequency than of the previous square-rooting circuits.

(iii) The proposed square-rooting circuits are very suitable for MOS IC technology.

(iv) The proposed square-rooting circuits provide a wide dynamic range.

(v) The proposed square-rooting circuits provide good temperature stability.

(vi) The paper is divided into four sections. Section II deals with the principle of operation of proposed circuits that employ MOS in subthreshold region. In section III, square-root and square domain MOS circuits based on generalized translinear principle are introduced. Section IV comprise of simulation results of proposed circuits followed by conclusion in section $\mathrm{V}$.

\section{Principle of Operation}

The first step in developing a translinear circuit is to identify the translinear loop. The loop contains an even multiple of forward biased junctions which are arranged in such a way that there are an equal number of clockwise facing and counterclockwise facing polarities as shown in Fig. 1. 


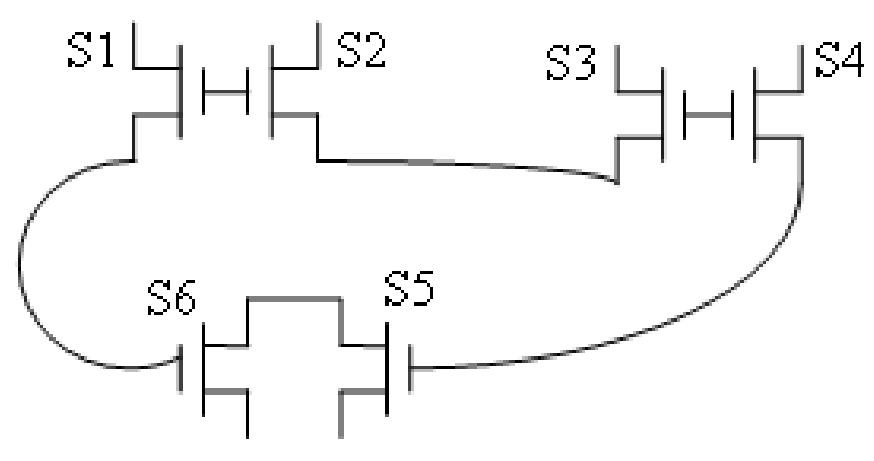

Fig.1. Translinear loop using MOS

The product of the current densities in the clockwise direction must be equal to the product of the current densities in the counterclockwise direction. For bipolar transistors, the principle is stated as

$$
\prod_{j \in c w} \frac{I_{j}}{A_{j}}=\prod_{j \in c c w} \frac{I_{j}}{A_{j}}
$$

where CW and CCW denotes, the set of indexes of clockwise facing junctions and the set of indexes of counterclockwise facing junctions respectively, $I_{j}$ is the collector current flowing in the $j^{\text {th }}$ transistor, and $A_{j}$ is the emitter junction area of the $j^{\text {th }}$ transistor. In [18], Seevinck and Wiegerink proposed a translinear principle for MOS referred as voltage translinear principle. The principle holds for loops of MOS transistors biased in strong inversion. It takes the form of

$$
\sum_{j \in c w} \sqrt{\frac{\boldsymbol{I}_{j}}{S_{j}}}=\sum_{j \in c c w} \sqrt{\frac{\boldsymbol{I}_{j}}{S_{j}}}
$$

where $S_{j}$ is the strength ratio, that is, $W / L$ ratio of the $j^{\text {th }}$ MOS transistor. However, MOS transistors operating in weak inversion [19] has an exponential current-voltage characteristic and can be considered as a translinear device in the sense that Gilbert originally intended. Such weak inversion MOS transistors can implement certain translinear circuits with fewer transistors as compared to bipolar. As discussed earlier, MOS was considered to be operating either in saturation mode or in weak inversion mode but when talking about moderate inversion then the current-voltage characteristic neither follows the exponential form nor the squarelaw form. In [20], Bradley A. Minch proposed a generalized translinear principle valid at all levels of inversion. Such principle takes the form of

$$
\prod_{j \in c w}^{N}\left(e^{\sqrt{I_{j} / s_{j} t_{s}}}-1\right)=\prod_{j \in c c w}^{N}\left(e^{\sqrt{I_{j} / s_{j} l_{s}}}-1\right)
$$

where $S_{j}$ and $I_{S}$ are the aspect ratio and specific current respectively, of the $j^{\text {th }}$ MOS transistor. The specific current is given by 
$I_{S}=2 \mu_{0} C_{o x} \frac{V_{t}^{2}}{k}$

For subthreshold MOS, $I_{j}<<S_{j} I_{s}$. Expanding exponential term of (6), the equation results in

$$
\begin{aligned}
& \prod_{j \in c w}^{j \in N}\left(1+\sqrt{\frac{I_{j}}{S_{j} I_{s}}}-1\right)=\prod_{j \in c c w}^{j \in N}\left(1+\sqrt{\frac{I_{j}}{S_{j} I_{s}}}-1\right) \\
& \prod_{j \in C w}^{j \in N} \sqrt{\frac{I_{j}}{S_{j} I_{s}}}=\prod_{j \in c c w}^{j \in N} \sqrt{\frac{I_{j}}{S_{j} I_{s}}}
\end{aligned}
$$

when each MOS transistor is assumed to be identical, their specific currents will be same. From (8)

$$
\begin{aligned}
& \prod_{j \in c w}^{j \in N} \sqrt{\frac{I_{j}}{S_{j}}}=\prod_{j \in c c w}^{j \in N} \sqrt{\frac{I_{j}}{S_{j}}} \\
& \prod_{j \in c w}^{j \in N} \frac{I_{j}}{S_{j}}=\prod_{j \in c c w}^{j \in N} \frac{I_{j}}{S_{j}}
\end{aligned}
$$

\section{Proposed Square-Root Circuit}

The architecture for performing square-root function is shown in Fig. 2. The four PMOS $M_{1}, M_{2}, M_{3}$ and $M_{4}$ act as translinear loop and rest of transistor, $M_{5}-M_{8}$ is configured as complex current mirror circuit. Each of the four PMOS transistors of TL is made to operate in subthreshold mode. Here, $I_{\text {in }}$ is the input current and corresponding output current $I_{\text {out }}$ is generated. $I_{\text {ref }}$ is the reference current.

$$
\frac{I_{3}}{S_{3}} \times \frac{I_{4}}{S_{4}}=\frac{I_{1}}{S_{1}} \times \frac{I_{2}}{S_{2}}
$$

Let each MOS in TL loop to have same strength ratio, i.e. $S_{1}=S_{2}=S_{3}=S_{4}$, equation (11) reduces to

$$
I_{3} \times I_{4}=I_{1} \times I_{2}
$$

For square-root circuit, $I_{3}=I_{4}=I_{\text {out }}, I_{1}=I_{\text {in }}$ and $I_{2}=I_{\text {ref }}$. Using (9)

$$
\begin{gathered}
I_{\text {out }} \times I_{\text {out }}=I_{\text {in }} \times I_{\text {ref }} \\
I_{\text {out }}=\sqrt{I_{\text {in }} \times I_{\text {ref }}}
\end{gathered}
$$




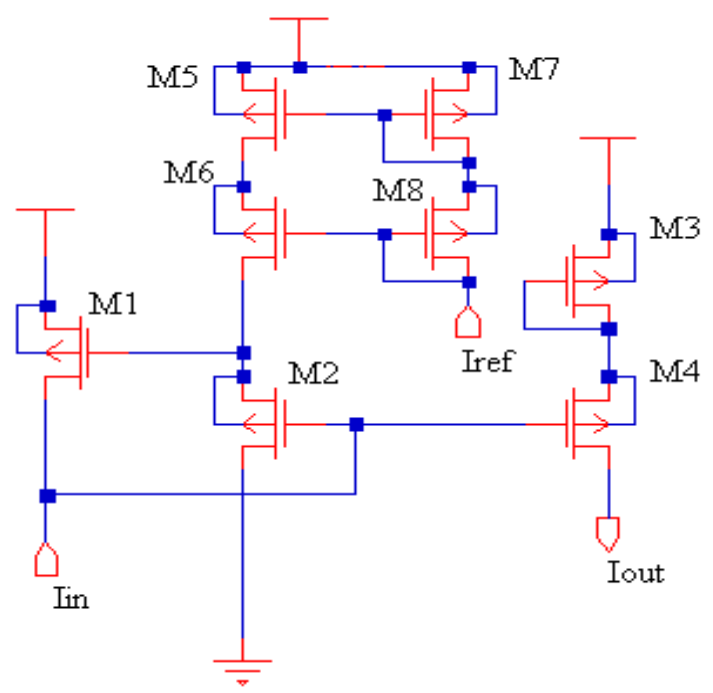

Fig.2. Square-root circuit

\section{Simulation Results}

The proposed current-mode square-root circuit is implemented using TSMC $0.18-\mu \mathrm{m}$ technology and the simulations were performed under normal condition (room temperature). The transistor's aspect ratio is summarized in table 1 . For the proposed square-root circuit, the input current $I_{\text {ref }}$ is constant dc current of value $1 \mathrm{nA}$ where the other input current $\mathrm{I}_{\mathrm{in}}$ is applied current as a triangular wave with amplitude of $100 \mathrm{nA}$ and its corresponding output $I_{\text {out }}$ is shown in Fig. 3. The simulated results when matched to theoretical value that is shown as $I_{\text {theoritical }}$ showed almost similar response.

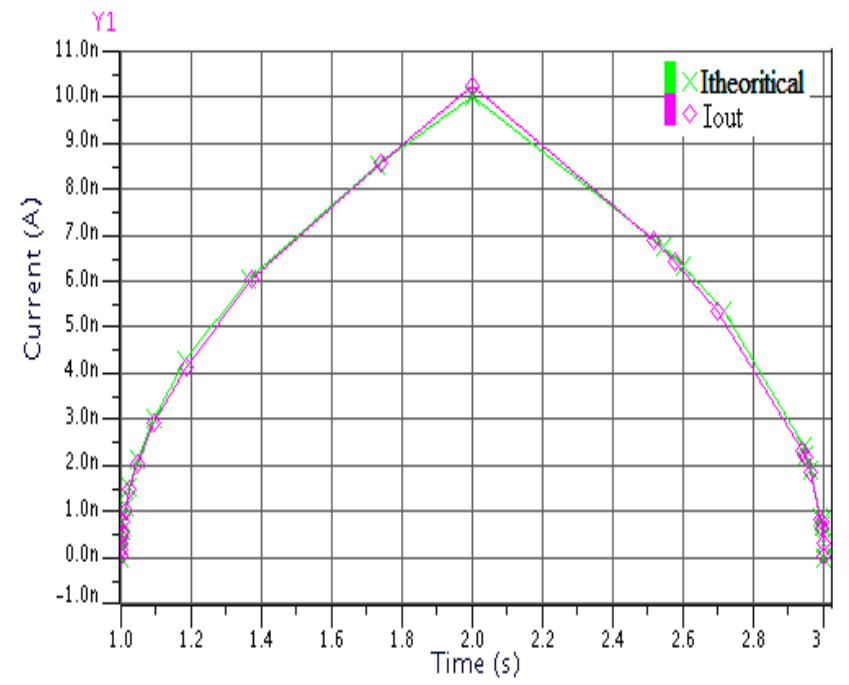

Fig.3. Simulation result of square-root circuit for $\operatorname{lin}=100 \mathrm{nA}$, Iref $=1 \mathrm{nA}$ 
Table 1.Transistor aspect ratios

\begin{tabular}{lll}
\hline & \multicolumn{2}{c}{ Square-root circuit } \\
\hline Transistors & $\mathrm{W}(\mu \mathrm{m})$ & $\mathrm{L}(\mu \mathrm{m})$ \\
& & \\
\hline M1 & 3.5 & 0.35 \\
M2 & 3.5 & 0.35 \\
M3 & 3.5 & 0.35 \\
M4 & 3.5 & 0.35 \\
M5 & 36 & 3.6 \\
M6 & 36 & 3.6 \\
M7 & 36 & 3.6 \\
M8 & 36 & 3.6 \\
\hline
\end{tabular}

\section{Conclusions}

This paper explored the approach of translinear principle based square-root circuit using MOSFET in subthreshold. The advantage of using such circuit is that it can be easily adjusted by the aspect ratios of MOS transistors to achieve high accuracy, which is more convenient than bipolar that is adjusted by the areas. In this paper, new simple square-rooting circuit based on translinear principle has been presented. Simulation results shown the proposed circuit provided wide input current range and good temperature stability. The proposed circuit can offer significant benefits in terms of signal processing. It can be useful in analysis of nonlinear distortion in CMOS log-domain filters. Such can also be used to develop an automatic gain-control (AGC) circuit as for designing of AGC, its gain is made to vary linearly with its control voltage.

\section{References}

[1] B. Gilbert, "Translinear Circuits: A Proposed classifications," Electronics Letters, vol. 11, no.1, pp.14-16, 1975.

[2] C. A. Mead, Analog VLSI and Neural Systems. AddisonWesley: Reading, MA, 1989.

[3] C.A. Mead, "Neuromorphic electronic systems," Proceedings IEEE, vol. 78, no. 10, pp. 1629-1636, Oct. 1990.

[4] E.A. Vittoz and J. Fellrath, "CMOS analog integrated circuits based on weak inversion operation," IEEE Journal of Solid-State Circuits, vol. 12, no. 3, pp. 224-231, June 1977.

[5] E. A. Vittoz, Micropower techniques, In Y. P. Tsividis and P. Antognetti, eds., VLSI Circuits for Telecommunications, Prentice Hall: 1985.

[6] A.G. Andreou and K. A. Boahen, "Synthetic neural circuits using current domain-signal representations," Neural Computation, vol. 1, pp. 489-501, 1989.

[7] A.G. Andreon, "Synthetic neural systems using current-mode circuits," Proceedings of the IEEE 1990 International Symposium on Circuits and Systems, New Orleans, pp. 2428-2432, May 1990.

[8] A.G. Andreou, Low power analog VLSI systems for sensory information processing, in B. Sheu, Edgar Sanchez-Sinencio and M. Ismail, eds., Microsystems Technologies for Multimedia Applications: An Introduction. IEEE Press: Los Alamitos, CA, 1995.

[9] K.A. Boahen, "Retinomorphic vision systems," Proceedings ofMicroNeuro-96. IEEE Computer Society Press: Los Alamitos, CA, 1996.

[10] E. O. Doebelin, Measurement Systems: Application and Design, New York: McGraw Hill, 2004.

[11] P. E. Allen and D. R. Holberg, CMOS Analog circuit design, Holt Rinehart and Winston, Inc., 1987.

[12] J. Millman and A. Grabel, Microelectronics, New York: McGraw-Hill, 1992. 
[13] I. M. Filanovsky and H. P. Baltes, Simple CMOS analog square-rooting and squaring circuits," IEEE Trans. Circuits and Systems-I, vol. 39, pp. 312-315, 1992.

[14] F.A. Khanday, C. Psychalinos, and N.A. Shah, "Universal filters of arbitrary order and type employing square-root-domain technique”. Int. J. Electronics, vol. 101, no. 7, pp. 894-918, 2014.

[15] A. Kircay, "Electronically tunable current-mode square-root domain first-order multifunction filter," Int. J. Electronics, vol.101, no. 2, pp. 212-219, 2014.

[16] S.-I. Liu, ISquare-rooting and vector summation circuits using current conveyors," IEE Proceedings. Circuits, Devices and Systems, vol. 142, 223226, 1995.

[17] W. Chiu, S.-I. Liu, H.-W. Tsao, and J.-J. Chen CMOS di®erential di®erence current conveyors and their applications," IEE Proceedings. Circuits, Devices and Systems, vol. 143, 91-96, 1996. 1151-1159.

[18] E. Seevinck and R. J.Wiegerink, "Generalized translinear circuit principle," IEEE Journal Solid-State Circuits, vol. 26, no. 8, pp. 1098-1102,Aug.1991.

[19] E. Vittoz and J. Fellrath, "CMOS analog integrated circuit based on weak-inversion operation," IEEE Journal Solid-State Circuits, vol. 12, no. 3, pp. 224-231, Jun. 1977.

[20] B. A. Minch, "MOS Translinear Principle for All Inversion Levels," IEEE Journal. Circuits and Systems, vol. 55, no. 2, pp. 121-125, 2008.

\section{Author's Profile}

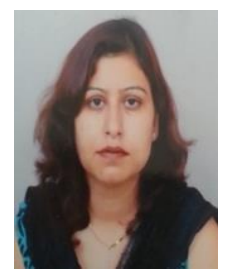

Bindu T. did her B. Tech from Vaish College (MDU), Rohtak, M.Tech from Lingyas College, Faridabad and is currently pursuing PhD in the area of VLSI from Ansal University. She is currently serving Ansal University as Assistant Professor in Electronics \& Communication Engineering department since five years. Earlier she has been associated with Dronacharya College, Gurgaon. Her research expertise includes Microprocessors, Analog Electronics, VHDL \& Digital Systems. She has always endeavored to introduce novel teaching ideas and methodologies, so that learning becomes interesting for students and they understand their subjects comprehensively.

How to cite this paper: Bindu Thakral,"Design of Low Power Current Mode Square-Root Circuit", International Journal of Engineering and Manufacturing(IJEM), Vol.5, No.4, pp.23-29, 2015.DOI: 10.5815/ijem.2015.04.03 